\title{
Representing Altered States of Consciousness in Computer Arts
}

\author{
Jonathan Weinel \\ Glyndŵr University \\ Plas Coch Campus, Mold Road, \\ Wrexham, LL112AW North Wales, UK \\ j.weinel@glyndwr.ac.uk
}

\begin{abstract}
It has been proposed that among the earliest known artworks produced by humans may have been representations of altered states of consciousness (ASCs). With the advent of modern computer technology that enables the creation of almost any sound or image imaginable, the possibility of representing the subjective visual and aural components of hallucinatory experiences with increased realism emerges. In order to consider how these representations could be created, this paper provides a discussion of existing work that represents ASCs. I commence by providing an overview of ASCs and a brief history of their use in culture. This provides the necessary background through which we may then consider the variety of art and music that represents ASCs, including: shamanic art and music, modern visual art, popular music, film and video games. Through discussion of the ways in which these examples represent ASC, a concept of 'ASC Simulation' is proposed, which emphasises realistic representations of ASCs. The paper concludes with a brief summary of several creative projects in computer music and arts that explore this area.
\end{abstract}

Altered states of consciousness. Psychedelic art. Visual music. Computer arts. Immersive technologies.

\section{INTRODUCTION}

In the 1920s, Heinrich Klüver carried out research regarding the effects of the hallucinogenic drug: mescaline. His research considered the underlying effects of mescaline on the visual system, which included the production of 'form constants': honeycomb, cobweb, funnel and spiral forms (Klüver 1971: 66). The research became influential in other fields such as anthropology, where it was suggested that these form constants might have provided a basis for early forms of rock art (LewisWilliams \& Dowson 1988). Lewis-Williams and Dowson's argument considers that the presence of forms reminiscent of the form constants in early rock art may suggest that those who created the artworks were experiencing these visual forms themselves during altered states of consciousness, and were therefore involved in shamanic practices. While the theory has been contested, the discussion is compelling since it highlights that representations of subjective visual experiences of hallucination may have been among the earliest subject matter for human artworks.

Moreover, in this paper we shall consider how altered states of consciousness (ASCs) have indeed provided a basis for many works of art and music in human culture since then. This discussion provides a background for us to then consider the ways in which computer artworks may be able to use the graphical and sound design capabilities to represent the visual and auditory components of subjective ASC experiences such as hallucination. We may therefore consider how computer graphics might enable us to actually create the types of visual patterns that people see during hallucinations, or how computer audio may enable us to design sounds similar to those that people hear during hallucinations.

While the focus of this paper will be on establishing the historic background and theory which supports my work in this area, I will also conclude by providing an overview of several creative projects in this area that explore approaches for representing altered states of consciousness in computer arts.

\section{ALTERED STATES OF CONSCIOUSNESS}

Altered states of consciousness (ASCs) are states such as dream, delirium or hallucination that fall outside of a commonly accepted 'normal consciousness'. The term 'altered state of consciousness' was notably used by Arnold Ludwig 
in Charles Tart's edited volume Altered States of Consciousness: A Book of Readings (1969). Ludwig discusses the various mechanisms that can produce ASCs, which include sensory deprivation, sensory overload, heightened states of attention and changes to body chemistry or neurophysiology. Specific examples of ASCs include sleep paralysis, hypnosis and trance states. Dreaming is sometimes considered as a form of ASC. Of particular interest for our discussion here, states of hallucination or altered awareness that may be produced by psychoactive drugs are also a form of ASC.

Various systems through which to consider the range of conscious states experienced by the individual have been proposed. For example, Fischer's cartography of ecstatic and meditative and states proposed that human consciousness can be considered in terms of an 'ergotropic' (states which promote expenditure of energy) and 'trophotropic' (states which promote conservation of energy) continuum (Fischer 1971). Considered in these terms, states of meditation or relaxation fall on one part of the continuum, while states such as ecstasy and hallucination occur on the opposing part.

Another conceptual approach to the range of consciousness states that people may experience is the 'state space' concept provided by Allan Hobson (2001: 44-45). This concept uses an 'activation, input, modulation' model of consciousness. 'Activation' describes the level of activity, which is high in waking states but low in states of unconsciousness or dreamless sleep. 'Input' describes the capacity for exchange of information via the external environment. The 'input' axis ranges from 'external' to 'internal' input; 'external input' refers to the reception of information from the external environment via senses such as sight and hearing. Conversely, 'internal input' refers to states in which sensory experiences may arise due to internal activity within the brain; for example, during states of dream or hallucination, visual images or sounds may be perceived which have no origin in the external environment. Lastly, the third axis of Hobson's model: 'Modulation' deals with the way in which the brain accesses and records events to memory, which may change in different states of consciousness.

Hobson's discussion of 'internal' and 'external' input sources is of particular relevance to our discussion, since we are interested in the way in which internal or subjective visual or auditory sensory experiences can be represented using artistic mediums or computers. For example, is it possible for a computer program to represent visual images that are similar to those experienced subjectively during a hallucination?

\section{A BRIEF HISTORY OF ASC IN CULTURE}

In order to consider this question, we shall first take a step back and provide a very brief historical overview of ASCs in culture; this will enable us to then consider the art and music which has been produced in the past which relates to ASCs. Such a discussion will enable us to consider underlying mechanisms for representing 'internal' sensory experience through artistic forms.

As Paul Devereux (2008) discusses, our ancestors have weaved an intricate history with the use of intoxicating plants and ASCs through ancient and pre-historic times. An interesting early example is that of the Neanderthal 'flower man' discovered by Ralph Solecki in 1975 at the Shanidar Cave in Northern Iraq. The man was discovered with several different types of plant material, which analysis showed contained pollen of plants that are known to have medicinal properties. Among these plants was Ephedra, a form of stimulant. Such a discovery may indicate that are far back as 60,000 years ago, early man may have been familiar with the capability of using plants for their consciousness altering properties.

Richard Evans Schultes, Albert Hofmann and Christian Rätsch (1996) provide a discussion of ninety-seven different types of psychoactive plants that are found across the globe, which have a history of use across various different cultures. Among these, Devereux discusses the possible Neolithic use of opium and/or cannabis at the $\mathrm{Er}$ Lannic and Gavrinis islands off the cost of Britanny; the use of opium in Bronze-Age Mycenaean and Minoan in the Mediterranean; and the use of opium in Egypt around 1500BC. He also refers to the suggestion that psilocybin mushrooms may have been a possible cause for the mushroom-headed figures of the Neolithic rock at found at the Tassili n'Ajjer Plateau in Africa, and the engravings found on razors at various Scandinavian sites.

Many of these early suggested uses of psychoactives are open to debate. One of the more enigmatic examples discussed by Devereux and Schultes, Hofmann and Rätsch is 'soma': the sacramental drink described in the sacred Aryan Rig Veda text. The text from around 3500 years ago in the Indus valley region (now Northern India, Afghanistan and Pakistan) described soma as having ecstatic mystical properties, which have convinced many that it was some form of hallucinogen; yet precisely what has remained a mystery, with theorists suggesting various psychoactive plants including Amanita muscaria mushrooms, Syrian rue or Stropharia cubensis mushrooms. 
Another contentious example of possible hallucinogen use is found in the Eleusinian mysteries of Ancient Greece. As Mircea Eliade (1979) discusses, the Eleusinian mysteries took place for nearly two thousand years around Athens and Eleusis, and centered around the legend of Demeter's daughter Persephone who was adducted by Pluto, lord of the underworld in Ancient Greek mythology. According to the legend, following a tumultuous period of attempting to reunite herself with her daughter, Demeter walked the earth and caused crops to stop growing on earth due to her grief. Eventually she persuaded Zeus to have his brother Pluto return Persephone, and they are reunited. However, before Demeter returns to the gods in Olympus, she taught the people of Eleusis her secret rites. These rites were the basis of the 'Eleusinian Mysteries' and were enacted twice each year. The larger of the two events involved a procession from Athens to Eleusis, during which a drink 'kykeon' was consumed. By the nighttime, the procession would arrive at Eleusis, where various rites and dances were enacted, and it is said that Persephone manifested amidst great flames. As Devereux discusses, such descriptions of the Mysteries have led some to suggest that the kykeon drink (which contained water, barley and pennyroyal), may have had hallucinogenic properties. The most compelling of these suggestions is that kykeon may have contained Ergot, a parasitic fungus which can grow on barley and contains similar alkaloids to LSD.

Beyond these early speculative examples, there are also many recorded examples from history of hallucinogens being used by various shamanic tribes. In such cases the full history of such practices is not fully known, but the first of these that became known to the modern world was the shamanism of reindeer-herder tribes found in Siberia, who used Amanita muscaria mushrooms. Indeed, as Devereux points out, the word 'shaman' derives from the Tungus word meaning 'to know'. Elsewhere, Pituri (a chewing tobacco narcotic) has been used by Aborigines in Australia, perhaps dating 40,000-60,000 years back; while Amanita muscaria and other plants have been used by shamans in Southeast Asia and the Pacific Islands. In Europe prior to the Reformation, witchcraft and 'wise women' were fairly widespread in the absence of modern medicine, and are known to have used various intoxicating plants and mushrooms (Harner 1973: 125-150). The Americas have a remarkably rich history of shamanic use of hallucinogenic plants, including Peyote use by the Native American Church; use of Peyote, Psilocybin mushrooms and Datura among indigenous peoples of Mexico such as the Huichol; and the use of Ayahuasca (a hallucinogenic brew) by peoples such as the Shipibo in the Peruvian Amazon of South America.
In modern Western culture, hallucinogens and other intoxicating substances are used, though as Devereux comments, the shift towards liquid intoxicants such as alcohol dates back as far as 2000 BC, and remains the dominant legal form of intoxication in many countries. Perhaps as a result of this, apart from various forms of neo-shamanism among hippies and new-age peoples, the use of psychoactive plants is more commonly associated with hedonistic lifestyles, rather than any specific religious use.

Of course, aside from hallucinogens we could also extend this discussion to consider other forms of naturally occurring ASC such as may occur in daily life, not least forms of dreaming or unconsciousness which most of us experience each day. Additionally, meditative practices are of note, as these have a long history of use among Buddhist, Hindu, Jewish, Christian and (more recently) atheist groups. When considering these additional forms of ASC, it should therefore be apparent that other forms of consciousness have a substantial history in human culture; little wonder then, that they have provided a basis for many works of art, literature and music. In the next section, a selective account of these will be provided.

\section{REPRESENTATIONS OF ASC IN ART AND MUSIC}

\subsection{Shamanic art and music}

As discussed, many of the historical examples where it is believed that cultures from the past may have been engaged in shamanic practices, are actually based on analysis of the art produced by those cultures. If we accept this analysis, the Upper-Paleolithic rock art discussed by LewisWilliams and Dowson; the patterns found on megaliths at the Gavrinis and Er Lannic sites; or the mushroom figures found on rock art at Tassili n'Ajjer, can be taken as examples of artworks that represent ASC. More specifically, the indication is that visual forms that are perceived subjectively during altered states of consciousness were used to inform the design of images in either a literal or metaphorical/symbolic sense.

Indeed, the visual art, textiles, jewellery and ornamental paraphernalia produced by modern-day shamanic societies can be viewed in similar terms, and in such cases it is easier to validate the meaning of the artworks since the artists are still living. The art of the Huichol peoples is discussed in Kathleen Berrin (1979), while Daniel Swan (1999) discusses the Peyote art produced by Native Americans. For both these groups, Peyote permeates their way of life in all aspects including 
their artwork. Thus a great deal of art relates symbolically to Peyote and/or their environment, as Peyote enables them to see it. While the work is often symbolic, some examples of brightly coloured patterns can be seen as literally reflecting the visual patterns seen during hallucinatory episodes.

Music is also used in shamanism in order to conduct the rituals, as documented in field recordings released on the Smithsonian Folkways label (Wasson 1957, Smith 1973). Gilbert Rouget (1985) has discussed the use of music in shamanism and in trance cultures such as those of Bali, where music plays a role in initiating a social situation that supports the production of trance states. While Rouget does not attribute any absolute capabilities for certain music to induce trance (it must be considered as part of a complex social structure that facilitates these states), he does hint at certain commonalities in music that enables the production of ASCs, such as quickening of tempos at key points in the ritual. Thus, it points towards the role of music as a device that communicates the social context, and carries certain affective properties that establish the mood of a situation within which trance can occur. In these terms it is reasonable to consider these musical forms less in terms of 'representations' of ASC, and more as communicative and affective tools that support the production and regulation of these states.

\subsection{Modern visual arts}

Psychedelic themes in visual arts are discussed by David Rubin (2010), and often exhibit similar principles of symbolic and/or literal representation as are found in shamanic art. Modern visual arts that align themselves with 'psychedelic' culture generally do so either by utilising aspects of the culture as subject matter, or incorporating conceptual views of 'consciousness expansion' into the work in some manner. Often this results in pieces that in a broad sense can be seen as symbolic or metaphorical representations of ASCs, or counter culture themes associated with them. However equally, many pieces also relate in a literal sense to the types of visual experience that may be subjectively perceived during a hallucination.

The latter of these approaches is of particular interest for our discussion. For example, we may consider Fred Tomaselli's Ripple Trees (1994). In this piece, the geometric patterns of the piece strongly relate to the actual types of visual images that one would expect to see during a hallucination, as discussed by Klüver and others. Considered in terms of Hobson's 'Input axis', the piece presents an 'internal' layer of visuals which relate to those that arise internally due to hallucination, while also superimposing these over an 'external' layer which represents what we presume to be a real-world environment. When considered in such terms, the piece can be viewed as a representation of subjective visual experience from a first-person perspective.

\subsection{Popular music and electronics}

The potential of recorded sound to represent ASCs can be considered by virtue of the fundamentally illusory quality of sound recordings. As Peter Doyle (2005) discusses, sound recording equipment fundamentally captures a trace or echo of an acoustic sound source. While in early devices such as Edison's phonograph, this was achieved by causing a stylus to vibrate and etch the sound wave into a rotating cylinder, in later devices the same principle is achieved by converting the sound vibration into an electrical voltage, and ultimately (in modern equipment) a digital signal. When these devices reproduce sound, they do so by reversing the process; allowing the recorded trace of the acoustic energy to indicate the movement of a diaphragm (such as a loudspeaker cone). This enables the listener to hear something that approximates the original sound.

However, while the original intention of sound recording was certainly to provide realism, as the technology evolved, engineers soon realised that equipment such as magnetic tape could be edited, in order to change the perceived reality of the original sound source. As Greg Milner (2009: 104128) discusses, radio shows could be pre-recorded to remove 'dead air' or mistakes. With the advent of multi-track recording, popular music quickly evolved into a complex mixture of recorded signals. Similarly development of electronic effects devices such as reverberation units, fuzz boxes, delays, flangers and others enabled signals to be altered and for certain spatial properties to be fabricated. When used on popular music recordings the overall effect was similar to that of a live performance by a group of musicians, but the recording could consist of takes from various points in time, and sound recorded from one or more positions. Therefore it was possible for the presented of reality within the recording to become increasingly stylised as popular music production techniques developed and fashions for different production styles evolved.

Admist this capability for sound recording to present an illusory version of reality, in the 1960s psychedelic rock music is particularly notable for exploiting the full range of potential for sound recording equipment to represent sound in a strange and wonderful ways that broadly connect with the concept of altered states of consciousness and psychedelic culture. A classic example is Tomorrow Never Knows by the Beatles; a piece 
which utilises a variety of tape loops and other studio processes to achieve a psychedelic impression.

Many other pieces of the era similarly represent altered states in various literal or symbolic ways; the use of sound manipulation can be taken either to reflect the perceptual distortions which accompany an altered state of consciousness, or can otherwise metaphorically reflect aspects of these states. For example, as Doyle (2005: 114117) discusses, reverb can metaphorically reflect a sense of the 'inner psyche', or the supernatural. Psychedelic pop music frequently exploits this potential in order to represent ASCs.

While these representations are common psychedelic music, in the live music created by artists such as the Grateful Dead, Hawkwind, or later on Psy-Trance (a form of electronic dance music), the music can also be seen as providing the necessary affective properties and cultural communication to support the induction of trancelike states. Indeed in relation to Psy-Trance this has been discussed by Graham St. John (2011), who indicates that modern electronic dance music festivals provide liminal spaces that facilitate trance. In general, this view considers electronic dance music as a sensory simulant, which in combination with lights, social context and drugs enables the production of ASCs, leading to transformation of the individual.

Taken in such terms, music is less a representation of ASC and more a vehicle for its induction. However, Psy-Trance music also contains many symbolic representations of ASCs, and uses of sound that could be viewed as directly reflecting perceptual distortions. Thus it functions on multiple levels, including the simulation of sensory distortions that occur during ASCs through the use of sound.

\subsection{Psychedelic Film}

Representations of altered states of consciousness in film typical combine a mixture of the visual and aurally based approaches that have been discussed so far. Jordan Belson was particularly interested in representing what he saw as work of the 'inner eye' using animated forms. His pieces such as the (unfinished) $L S D$ (1962) can be seen in terms of the form constants discussed by Klüver, as William Wees discusses (1992). Visual music in general adopts a synesthetic approach that seems to be highly suited to 'psychedelic' interpretations, perhaps because hallucinatory states themselves promote synesthetic perception.

Perhaps not surprisingly then, similar approaches to those found in visual music and experimental cinema were adopted to create the psychedelic light shows of 1960s acid rock gigs. These in turn later evolved into the early computer visualisations of VJ culture, as seen in the $X$-Mix series, or in Jeff Minter's Atari ST programmes such as Trip-A-Tron. The burgeoning VJ culture of the 1990s can frequently be seen in terms of representing the hallucinatory patterns seen during LSD, as representations of a an LSD trip in 3D graphics, or as reflecting the visual effects of hallucinogens on colour perception. When seen in such terms early virtual reality technology can be seen as part of the 'cyberdelic' nexus discussed by Timothy Leary, Terrance McKenna and through the cyberpunk fiction of William Gibson. This view of virtual reality and computer technology broadly considers the potential of these new technologies to transform human consciousness in variety of ways, and was influential on a lot of the electronic dance music and VJ culture of this period.

Of course, Hollywood has had its fair share of psychedelic scenes too, notably in movies such as Ken Russell's Altered States (1980), or Terry Gilliam's Fear and Loathing in Las Vegas (1998). The former movie was based on John Lilly's research regarding the hallucinatory effects of sensory isolation tanks, while the latter movie adapts Hunter S. Thompson's classic 'gonzo journalism' novel. The actual representations of hallucination in these films draw upon both symbolic/metaphorical approaches as well as literal ones. For example, the hallucination scenes of Altered States are heavily laden with surrealistic imagery, as are those in Fear \& Loathing in Las Vegas. However the firework impressions of Altered States also hint at a more literal representation of the sensory experience of visual hallucinations, while Fear \& Loathing in Las Vegas contains many computer graphics effects that enable textures to flow and colours to pulsate in a manner consistent with what people actually describe during experiences of hallucination. Of course, these are works of fiction aimed at entertainment, so realism is not particularly important, yet nonetheless, aspects of these representations are grounded to some degree in what people might really experience during an ASC.

\subsection{ASC Video Games}

In recent years there has been an increase in representations of intoxicated states in video games. While there are early examples of intoxication in games, most are fairly simplistic. For example, in Fantasy World Dizzy (1989) the player character can consume alcoholic spirits, which cause him to roll around drunkenly. In more modern titles, the increase in graphical capabilities, sound and realism provided in games such as first- 
person shooters enables much more sophisticated representations of intoxicated states. As with movies, the approaches are often symbolic/ metaphorical but occasionally utilise some literal/realistic approaches.

For example, in Grand Theft Auto V (2013), one scene features various graphical blurring effects and slowing of speech effects to represent the onset of a drug experience. This is followed by a representation of the game character falling from the sky with various bright graphical augmentations which blur the visuals and increase the contrast. While the scene is a parody, some of these effects do connect with the changes in visual experience or the types of hallucination that are described in studies that investigate experiences of hallucination. Similarly, the Far Cry 3 (2012) 'Mushrooms in the Deep' episode features a combination of animated camera properties, visual effects and sound in order to reflect a hallucinatory state that perhaps is the most realistic seen in a modern computer game to date.

\section{TOWARDS A THEORY OF ASC SIMULATION}

Through the course of this paper we have considered what is meant by ASCs, their prevalence in culture, and some examples from art and music that seeks to represent them. Of particular interest in recent examples from popular music, visual music, VJ culture and video games, are the ways in which artists have been able to provide representations of ASC that hint at the real, subjective experience of these states (as opposed to more metaphorical or symbolic representations). Following this discussion we may therefore consider a general approach for providing more realistic representations of ASC, which could be considered to approach something we might consider simulation.

Such an approach can be considered in terms of simulating the subjective auditory and visual components of sensory experience, using sound and computer graphics. Indeed, first-person perspective computer games already simulate human perception, by using a camera to represent sight, and stereo audio to represent hearing of a virtual character. We can consider developing this approach in order to consider the use of computer graphics and sound in order to represent the subjective experience of hallucination in a fairly realistic way. Notably, while various metaphorical or symbolic modes of representation are possible, in order to achieve realism these should be avoided in favor of a more literal approach, which translates visual or aural experiences of hallucination into their visual or aural equivalents in computer graphics and sound.
The way in which these 'ASC Simulations' can be designed may be usefully considered in terms of Hobson's 'Input Axis'. Thus, the way in which 'external' sensory input is represented can be modified in accordance with research regarding the changes to visual and aural sensory experience that are produced during a hallucination. Similarly. 'internal' sensory input; the geometric patterns, auditory hallucinations or other such phenomena can be designed based on what the research has to say about the contents of these experiences.

\section{PROJECTS}

In this section I shall outline several projects within the field of computer music and arts that I have produced over the last few years that relate to the concepts discussed. These are discussed in summary; for more information please visit my website (www.jonweinel.com).

\subsection{Entoptic Phenomena in Audio}

This project grew out of my PhD work in musical composition that explored the use of altered states of consciousness as a basis for composing electroacoustic music. The pieces explore various approaches through which the concept of hallucination informs the design of musical materials and structure. Notably on the piece Entoptic Phenomena, the structure of the music closely follows that of a hallucination. The vinyl record presentation of the music features a painting by myself that is based upon Klüver's form constants (Weinel 2014).
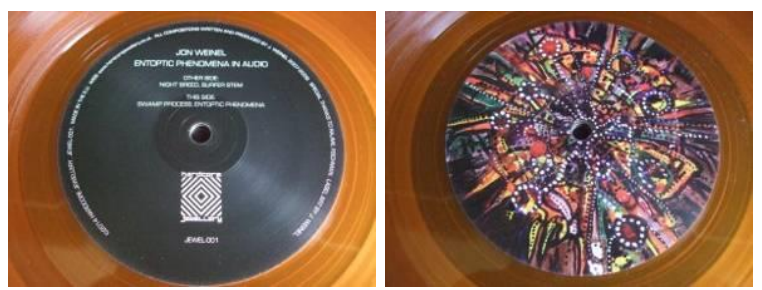

Figure 1: Entoptic Phenomena in Audio vinyl

\subsection{Optical Research}

Optical Research is a curated collection of works by various artists working in visual music, experimental film and associated forms. Many of these pieces can be considered as 'synesthetic', and a few relate directly to the concept of altered states of consciousness. For example, my piece Mezcal Animations, uses direct animation and electroacoustic sound to explore the concept of hallucinations, while Brummbaer's piece Where in the Universe is Timothy Leary? presents a poetic journey through a fractal universe, paying homage to Timothy Leary. 


\subsection{Psych Dome}

Psych Dome (Figure 2) is an interactive installation which uses a NeuroSky Mindwave headset as a controller for real-time audio-visual algorithms created in Processing and Max/MSP. The brainwaves provided by the headset (which is a consumer-grade EEG device) are used as a control signals to affect parameters of the visual and sound processes. The visual processes are based on Klüver's form constants, and enable animated geometric tunnel and spiral forms to be produced, while the sound produces an aural accompaniment for these. Aspects such as the colours of the visualisation or the pitches of the sounds change with each iteration of the artwork depending on the signals from the headset.

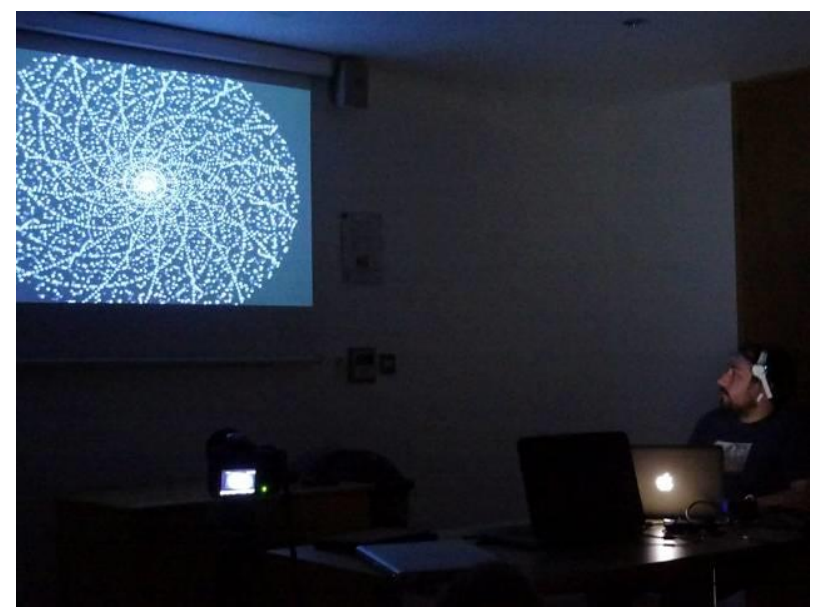

Figure 2: Psych Dome demonstration at EVA London 2014. A consumer-grade EEG device is used to control parameters of real-time visuals and sound generation (Photo @ Kia Ng 2014)

\subsection{Quake Delirium}

A modification for the game Quake written in Max/MSP, Quake Delirium (Figure 3) affects the game by automating graphical and game parameters that are usually static. This enables various visual properties of the game to change in time, reflecting aspects of hallucination. A supporting soundtrack is also provided.

Recently this project was updated to create Quake Delirium EEG, a version of the system which substitutes the automation of certain colour parameters for real-time control signals provided by the NeuroSky MindWave headset, in a similar manner to Psych Dome. The project thereby explores the concept of user interactivity in an early biofeedback driven 'ASC Simulation'.

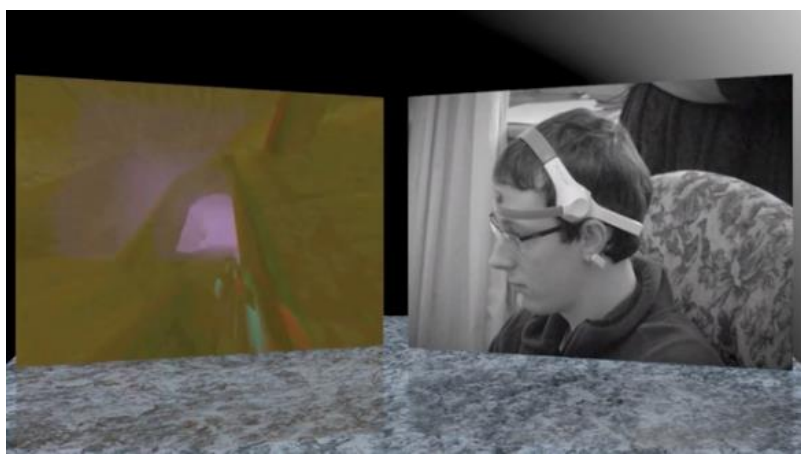

Figure 3: Quake Delirium EEG uses a NeuroSky MindWave headset to control graphical parameters of a modified video game

\subsection{Unity ASC Simulation}

This on-going project is currently in the early stages, but seeks to use the Unity game engine as a basis for designing an ASC Simulation. The current version contains several key mechanisms. Firstly, it attenuates amplitude of objects within the game environment that are the focus of the game character's attention. Attention is based on the object the character is looking at (i.e. in the centre of the screen). When an object is the focus of attention in the visual field, all other sound levels are reduced. The concept of attenuating unattended sources relates to the theory of attention developed by Anne Treisman (discussed in John Driver 2001). Here it is interpreted to indicate audio design, and thereby reflect the concept of increased focus of attention on a particular object that can become enhanced during an ASC.

Additionally, the project also enables a parameter that 'enhances' sound, which functions by increasing the brightness and high-frequency content of the sounds. This reflects the subjective experience of sounds becoming more interesting, bright or enjoyable which is sometimes described in accounts of ASC (Weinel et al. 2014).

The project is on-going and it is hoped to incorporate further specific mechanisms that reflect the auditory or visual changes that may occur during a hallucination, through the medium of a first person perspective 3D game.

\section{CONCLUSION}

In this paper I have focused on the historical background that informs my work in computer arts regarding ASCs. In particular, I have discussed a definition of ASCs based on Hobson's 'AIM' model, the 'Input axis' of which usefully informs my work in this area. Altered states of consciousness have historically been pervasive in human culture across the globe, and in many cases have formed the 
basis for works of art and music. While many of these artworks use symbolic or metaphorical modes of representation, even early rock art examples can be considered as literal representations of the subjective 'internal' visual experience of hallucination in some respects. It is these that are currently of particular interest for my work, as we may conceive of using computer graphics in combination with immersive technologies and biofeedback in order to provide increasingly realistic interactive 'ASC Simulations'. While there are various possible applications for these 'ASC Simulations' ranging from therapeutic purposes to entertainment, perhaps the most compelling reason why we should seek to devise the simulations is the same which has often driven human interest in ASCs of millennia: philosophical enquiry into the nature and meaning of our conscious experience.

\section{ACKNOWLEDGEMENTS}

The Entoptic Phenomena in Audio project relates to my PhD work that was supported by my supervisor Rajmil Fischman, my colleagues at Keele University, and an AHRC grant. Optical Research includes contributions by Ryo Ikeshiro, Josh Simmons, Ocusonic, Benjamin Rowley, Maxime Corbeil-Perron, Diego Garro, Mark Pilkington, Richard Scott, Scott Kiernan, Diego Capoccitti, VJ Chaotic, Vibrasphere and Brummbaer. The Psych Dome and Quake Delirium projects were also used as a basis for user-testing studies that explored human-computer interaction issues. These were carried out in collaboration with my colleagues at Glyndŵr University: Stuart Cunningham, Nathan Roberts, Darryl Griffiths and Shaun Roberts.

\section{REFERENCES}

Berrin, K. (1979) Art of the Huichol Indians. Harry N. Abrams, Inc.

Devereux, P. (2008) The Long Trip: A Prehistory of Psychedelia. Brisbane: Daily Grail.

Doyle, P. (2005) Echo and Reverb: Fabricating Space in Popular Music Recording, 1900-1960. Wesleyan University Press.

Driver, J. (2001) A selective review of selective attention research from the past century, British Journal of Psychology, 92. 53-78.

Eliade, M. (1979) The Eleusinian Mysteries. In $A$ History of Religious Ideas: Vol.1 From the Stone Age to the Eleusinian Mysteries. Chicago: William Collins Sons \& Co.

Fischer, R. (1971) A Cartograph of Ecstatic and Meditative States. Leonardo 174, 59-66.
Harner, M. (1973) Hallucinogens and Shamanism. Oxford University Press.

Hobson, A. (2001) The Dream Drugstore: Chemically Altered States of Consciousness. MIT Press.

Klüver, H. (1971) Mescal and Mechanisms of Hallucinations. University of Chicago Press.

Lewis-Williams, J. D. \& Dowson, T.A. (1988) The Signs of All Times: Entoptic Phenomena in Upper Palaeolithic Art. Current Anthropology, 29, 201245.

Ludwig, A. M. (1969) Altered States of Consciousness. In C. Tart (ed.), Altered States of Consciousness: A Book of Readings. Wiley.

Milner, G. (2009) Perfecting Sound Forever. Granta Books.

Rouget, G. (1985) Music and Trance: A Theory of the Relations Between Music and Possession. University of Chicago Press.

Rubin, D. (2010) Psychedelic: Optical and Visionary Art Since the 1960s. MIT Press.

Schultes, R. E. Hofmann, A., \& Ratsch, C. (1996) Plants of the Gods: Their Sacred, Healing and Hallucinogenic Powers, 2nd edition. Healing Arts Press.

Smith, H. E. (1973) Kiowa Peyote Meeting [CDr]. Folkways Records.

St. John, G. (2011) DJ Goa Gil: Kalifornian Exile, Dark Yogi and Dreaded Anomaly. Dancecult: Journal of Electronic and Dance Music Culture 3(1).

Swan, D. C. (1999) Peyote Religious Art: Symbols of Faith and Belief. University Press of Mississippi.

Wasson, R. G. \& Wasson, V. P. (1957) Mushroom Ceremony of the Mazatec Indians of Mexico [LP]. Folkways Records.

Wees, W. C. (1992) Making Films for the Inner Eye: Jordan Belson, James Whitney, Paul Sharits. In Light Moving in Time: Studies in the Visual Aesthetics of Avant-garde Film. University of California Press.

Weinel, J. (2014) Entoptic Phenomena in Audio [12" Vinyl]. Hardcore Jewellery, JEWEL-001. Available from: http://www.hardcorejewellery.co.uk

Weinel, J. Cunningham, S. \& Griffiths, D. (2014) Sound Through The Rabbit Hole: Sound Design Based On Reports of Auditory Hallucination. ACM Proceedings of Audio Mostly 2014, Aalborg University, Denmark. 